\title{
Characteristics of Dapagliflozin Responders: A Longitudinal, Prospective, Nationwide Dapagliflozin Surveillance Study in Korea
}

\author{
Eugene Han · Ari Kim · Sung Jae Lee · Je-Yon Kim • Jae Hyeon Kim • \\ Woo Je Lee · Byung-Wan Lee
}

Received: February 22, 2018 / Published online: July 11, 2018

(C) The Author(s) 2018

\begin{abstract}
Introduction: Sodium glucose co-transporter 2 (SGLT2) inhibitors, such as dapagliflozin, have demonstrated favorable effects in patients with type 2 diabetes (T2D). However, there are limited reports in the literature regarding the glucose-lowering effects of SGLT2 inhibitors in actual clinical settings.

Methods: The post-marketing surveillance data from a longitudinal prospective study of 2007 patients with T2D who were prescribed dapagliflozin (10 mg/day) were analyzed (ClinicalTrials.gov, NCT02252224).
\end{abstract}

Enhanced digital features To view enhanced digital features for this article go to https://doi.org/10.6084/ m9.figshare.6736496.

Electronic supplementary material The online version of this article (https://doi.org/10.1007/s13300018-0470-9) contains supplementary material, which is available to authorized users.

E. Han

Division of Endocrinology, Department of Internal Medicine, Keimyung University School of Medicine, Daegu, Korea

A. Kim · S. J. Lee · J.-Y. Kim

AstraZeneca, Seoul, Korea

J. H. Kim

Division of Endocrinology and Metabolism, Department of Medicine, Sungkyunkwan University School of Medicine, Seoul, Korea
Results: After 12 weeks of dapagliflozin treatment, glycated hemoglobin (HbA1c) and body mass index were significantly decreased $(P<0.001)$ from $8.1 \pm 1.3 \%$ to $7.5 \pm 1.2 \%$ and from $28.1 \pm 4.4$ to $27.6 \pm 4.2 \mathrm{~kg} / \mathrm{m}^{2}$, respectively. Both body weight and HbA1c were reduced in $67.7 \%$ of patients, and $\mathrm{HbA1c}$ was lowered in $75.1 \%$. Younger age, male sex, shorter diabetes duration, higher baseline HbA1c and estimated glomerular filtration rate (eGFR), and having dapagliflozin as add-on therapy were associated with stronger HbA1c reductions after dapagliflozin use (all $P<0.05$ ). Moreover, subgroup analysis of eGFR of subjects with renal hyperfiltration (eGFR $\geq 120 \mathrm{ml} / \mathrm{min} /$ $1.73 \mathrm{~m}^{2}$ ) showed the largest reduction in glucose level (\% change, $-9.5 ; 95 \%$ CI -6.8 to -12.3 for HbA1c; $P<0.001)$. Multivariable logistic regression analysis showed that recent T2D diagnosis and higher HbA1c at baseline in patients who received an add-on regimen of dapagliflozin were statistically significantly

\footnotetext{
W. J. Lee $(\bowtie)$

Division of Endocrinology and Metabolism, Department of Internal Medicine, University of Ulsan College of Medicine, Seoul, Korea e-mail: lwjatlas@amc.seoul.kr

B.-W. Lee $(\bowtie)$

Division of Endocrinology, Department of Internal Medicine, Yonsei University College of Medicine, Seoul, Korea

e-mail: bwanlee@yuhs.ac
} 
associated with a dapagliflozin response (all $P<0.05)$.

Conclusions: Dapagliflozin provides benefits for glycemic control and body weight. Patients in a relatively early stage of the course of diabetes with renal hyperfiltration might be more suitable for and gain maximal benefit from dapagliflozin treatment.

Trial Registration: ClinicalTrials.gov identifier, NCT02252224.

Funding: AstraZeneca.

Keywords: Clinical trials; Nephropathy; Obesity; Pathophysiology; SGLT2 inhibitors

\section{INTRODUCTION}

Sodium glucose co-transport 2 (SGLT2) is a member of a family of ATP-dependent proteins located in the $S 1$ segment of the proximal renal tubules and manages up to $90 \%$ of the filtered glucose reuptake by the concentration gradient [1]. Inhibitors of SGLT2 comprise a novel class of anti-diabetic agents that has been gaining attention because of their unique mode of action and effectiveness in lowering blood glucose and reducing body weight independently of insulin [2, 3]. In addition, recent studies demonstrate that SGLT2 inhibitors have beneficial effects on cardiovascular disease-related mortality, blood pressure [4], hepatic steatosis [5], and visceral fat mass [6].

Dapagliflozin is an SGLT2 inhibitor with anti-hyperglycemic activity. Dapagliflozin monotherapy reduces an average of $0.9 \%$ glycated hemoglobin (HbA1c) and decreases body weight by up to $3 \mathrm{~kg}$ in randomized controlled trials (RCT) [7]. In combination with other antidiabetic medications, dapagliflozin provides sustained glycemic control and body weight reduction $[2,8]$. Compared with metformin, dapagliflozin therapy is superior in lowering blood glucose [9]. With lower hypoglycemia risk and high tolerability, dapagliflozin appears to fulfill some unmet needs in diabetes management [2].

Despite previous clinical studies demonstrating the favorable effectiveness of dapagliflozin, there are limited reports on the effectiveness of SGLT2 inhibitors in actual clinical practice settings, especially in Korea. Furthermore, the characteristics of patients who respond to SGLT2 inhibitors have not been fully determined. Thus, the aim of this study was to investigate the characteristics of dapagliflozin responders and evaluate the association between the glucose-lowering effectiveness of dapagliflozin and other clinical parameters in an actual clinical setting.

\section{METHODS}

\section{Design and Population}

This investigation was a non-comparative, prospective, non-interventional, regulatory post-marketing surveillance (PMS) study (ClinicalTrials.gov, no. NCT02252224) designed to evaluate the safety and effectiveness of dapagliflozin (Forxiga ${ }^{\circledR}, 10 \mathrm{mg}$ ) in patients with type 2 diabetes mellitus (T2D). A total of 3371 T2D patients from 123 medical facilities across Korea who were being treated with dapagliflozin were recruited from September 2014 to November 2016. Patients were recruited to be followed for either 12 weeks (maximum 16 weeks) or 24 weeks (maximum 30 weeks). Patients followed for 12 weeks accounted for the greater part of the study population, as only $10 \%$ of the total recruited patients at 12 weeks were mandatorily required to have long-term followup by the regulatory authority in Korea. For the initial study design, all patients who had taken at least one pill were included $(n=3371)$. However, a total of 1364 patients were excluded from analysis, as shown in Fig. 1. Only 2007 patients showed competent adherence to dapagliflozin therapy and were included in the effectiveness analysis at 12 weeks.

This study was conducted in compliance with the ethical principles of the "Basic Standards for the Re-examination of New Medicines" published by the Ministry of Food and Drug Safety in Korea. Approval from the Institutional Review Board was obtained according to the rules of each institution as required for a PMS. All procedures followed were in accordance with the ethical standards of the 


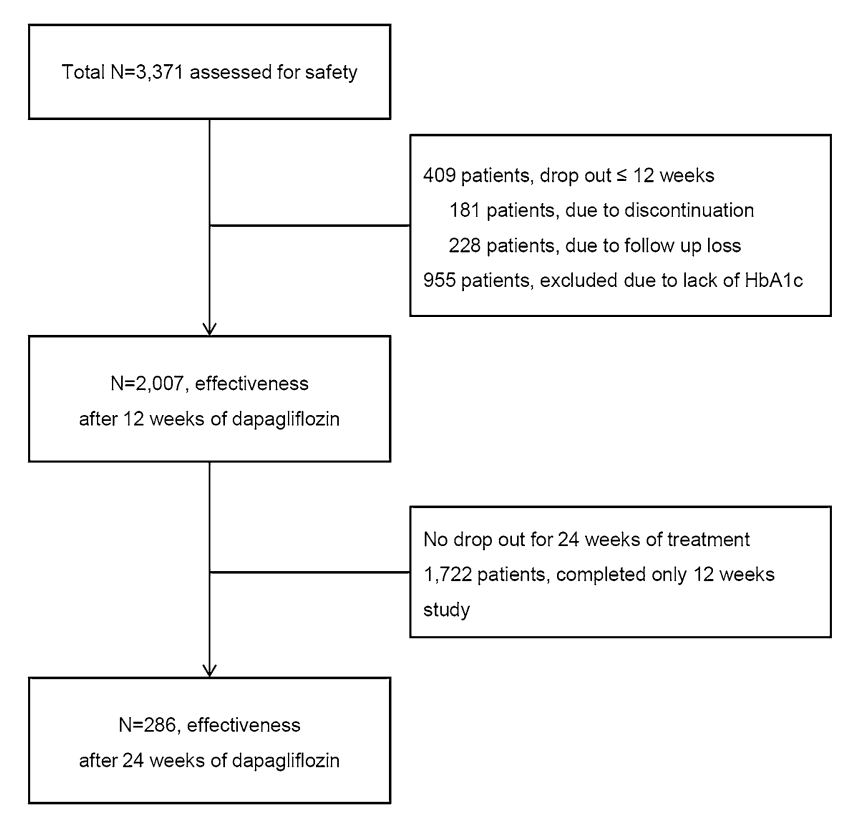

Fig. 1 Study flow diagram demonstrating analysis population. Post-marketing surveillance study designed to evaluate the safety and effectiveness of dapagliflozin

responsible committee on human experimentation (institutional and national) and with the Helsinki Declaration of 1964, as revised in 2013. Informed consent was obtained from all patients for being included in the study.

The patient population for this study consisted of Korean patients who were at least 18 years of age, had been diagnosed with T2D, and could start dapagliflozin therapy according to a reasonable indication for treatment in an ambulatory care setting. Patients were treated by accredited physicians according to routine practices at Korean healthcare centers throughout the study. After receiving written informed consent from the potential participants, each survey physician enrolled the patients who were receiving dapagliflozin for the first time and who had received at least one dose. Each study participant was followed until the observational periods were completed.

Exclusion criteria included patients who were treated with dapagliflozin outside of clinical relevance in Korea or who were contraindicated for the use of dapagliflozin. Contraindications included (1) patients who had a history of hypersensitivity to the active substance or to any of the excipients, (2) type 1 diabetes mellitus or diabetic ketoacidosis patients, (3) patients with hereditary problems of galactose intolerance, lactase deficiency, or glucose-galactose malabsorption, and (4) patients with severe renal impairment (estimated glomerular filtration rate [eGFR] $<30 \mathrm{ml} /$ $\min / 1.73 \mathrm{~m}^{2}$, calculated using the Chronic Kidney Disease Epidemiology Collaboration equation [10]), with end-stage renal disease, or on dialysis. Other anti-diabetic medications used by patients were maintained, titrated, or switched in accordance with the physician's decision during the periods of dapagliflozin treatment. Lifestyle modification therapy and education for both diet and exercise were also provided in parallel with medications.

\section{Effectiveness Assessment}

The comparable variables for the effectiveness of dapagliflozin after 12 and 24 weeks of treatment were changes in HbA1c, fasting plasma glucose (FPG), postprandial glucose (PPG), body mass index (BMI), and blood pressure. For PPG, patients underwent a conventional 2-h oral glucose tolerance test at baseline, 12 and 24 weeks (in case of long-term surveillance). Analysis of the effectiveness of dapagliflozin was conducted 
using stratified categorization with the responsiveness for the dapagliflozin treatment. Based on a previous report [11], we arbitrarily defined the responders as those exhibiting $a \geq 10 \%$ reduction in $\mathrm{HbA} 1 \mathrm{c}$ values after 12 weeks of dapagliflozin treatment. The relative change $(\Delta)$ was calculated as [(baseline value) - (post-dapagliflozin treatment value $(\geq 12$ weeks) $)] /$ (baseline value $) \times 100$ (\%). The absolute $\Delta$ was calculated as [(baseline value) - (post-dapagliflozin treatment value $(\geq 12$ weeks))].

\section{Safety Assessment}

Patients participating in the study were excluded or withdrawn from the continuation of treatment or the study for the following reasons: loss of follow-up information, adverse events, patient's decision, or physician's decision. If a patient discontinued the treatment during the study, the patient was contacted to collect all information regarding adverse events and the reasons why they wanted to stop the treatment. Patients who discontinued the study were included in the safety population if they had taken at least one dose of dapagliflozin. Adverse events and serious adverse events recorded in the electronic case report forms were recoded and reanalyzed according to the current routine classification [12].

\section{Statistical Analysis}

Data are presented as the mean \pm standard deviation for continuous variables and as number or percentage for categorical variables. The simple changes of blood pressure, glycemic and lipid parameters after dapagliflozin treatment were compared using a paired $t$ test. For effectiveness analysis, missing values were imputed using the last observation carried forward method. A mixed effect model repeated measure test among three visits (baseline, $\geq 12$ and $\leq 24$ weeks, and $\geq 24$ weeks) was performed only if each variable was measured at $>24$ weeks. The receiver-operating characteristic (ROC) curve was also used for calculating the cutoff value for the relative change expectation of HbA1c levels $>10 \%$ from the baseline. Three groups were compared by one- way analysis of variance (ANOVA) and Bonferroni tests in terms of changes from the baselines for detailed analysis according to eGFR. Scatter graphs were rendered and correlation coefficients calculated to confirm the correlation between changes of HbA1c and body weight or between relative HbA1c changes and baseline HbA1c. The relative factors for dapagliflozin response were obtained using univariate and multiple logistic regressions. Risk is reported in the form of odds ratios (ORs) and 95\% confidence intervals (CIs). All statistical analyses were performed using SAS (version 9.4, SAS Institute, Cary, NC, USA) for the calculations and NCSS (version 11, NCSS Co., Kaysville, UT, USA) for the graphics. $P$ values $<0.05$ were considered statistically significant.

\section{RESULTS}

\section{Baseline Characteristics by Study Population}

A total of 2007 individuals with T2D were analyzed (Fig. 1). Patient baseline characteristics are shown in Table 1 . The mean age of the study population was 52.8 years, and $51.1 \%$ were female. The mean BMI was $28.1 \mathrm{~kg} / \mathrm{m}^{2}$ and mean waist circumference was $92.2 \mathrm{~cm}$. According to the definition of obesity in the Asian Pacific Region Guideline (BMI $\geq 25 \mathrm{~kg}$ / $\mathrm{m}^{2}$ ) [13], $76.2 \%$ of the patients in this study were obese. Although the duration of T2D was relatively short (median 26.0 months), $22.3 \%$ of the patients reported having T2D-related complications. Dapagliflozin treatment was offered as switch medication in $46.6 \%$ participants and as add-on therapy in $53.3 \%$. Over $90 \%$ of the study population was prescribed metformin at baseline. The baseline mean FPG, PPG, and $\mathrm{HbA1c}$ were $166.2 \mathrm{mg} / \mathrm{dl}, 233.1 \mathrm{mg} / \mathrm{dl}$, and $8.1 \%$, respectively.

\section{Effectiveness of Dapagliflozin on Glucose, Body Weight, and Blood Pressure}

After 12 weeks of treatment with dapagliflozin, HbA1c, FPG, and PPG were statistically significantly $(P<0.001)$ lowered (from $8.1 \pm 1.3 \%$ to 
Table 1 Baseline characteristics of study population

\begin{tabular}{|c|c|}
\hline Baseline parameter & Value \\
\hline Age (years); mean (SD) & $52.8(11.1)$ \\
\hline Age $\geq 50$ years, $N(\%)$ & $1296(64.6)$ \\
\hline \multicolumn{2}{|l|}{ Sex, $N(\%)$} \\
\hline Male & $982(48.9)$ \\
\hline Female & $1025(51.1)$ \\
\hline Diabetes duration, months (IQR) & $26.0(3.9-65.6)$ \\
\hline$\geq 24$ months, $N(\%)$ & $472(23.5)$ \\
\hline Body weight, kg (SD) & $75.8(15.0)$ \\
\hline BMI, $\mathrm{kg} / \mathrm{m}^{2}(\mathrm{SD})$ & $28.1(4.4)$ \\
\hline Obesity $\left(\geq 25 \mathrm{~kg} / \mathrm{m}^{2}\right), N(\%)$ & $1092(76.2)$ \\
\hline Waist circumference, cm (SD) & $92.2(10.2)$ \\
\hline $\mathrm{SBP}, \mathrm{mmHg}(\mathrm{SD})$ & $128.1(14.2)$ \\
\hline $\mathrm{DBP}, \mathrm{mmHg}(\mathrm{SD})$ & $77.8(10.3)$ \\
\hline $\mathrm{FPG}, \mathrm{mg} / \mathrm{dl}(\mathrm{SD})$ & $166.2(48.8)$ \\
\hline PPG, mg/dl (SD) & $233.1(80.4)$ \\
\hline HbAlc, \% (SD) & $8.1(1.3)$ \\
\hline Serum creatinine $(\mathrm{mg} / \mathrm{dl})$ & $0.8(0.2)$ \\
\hline $\mathrm{eGFR}, \mathrm{ml} / \mathrm{min} / 1.73 \mathrm{~m}^{2}(\mathrm{SD})$ & $95.4 \pm 15.6$ \\
\hline \multicolumn{2}{|c|}{ Diabetes-related complications, $N(\%)$} \\
\hline No & $1559(77.7)$ \\
\hline Yes & $448(22.3)$ \\
\hline \multicolumn{2}{|l|}{ Manner of dapagliflozin use, $N(\%)$} \\
\hline Add-on & $1069(53.3)$ \\
\hline Switch & $936(46.6)$ \\
\hline Unknown & $2(0.1)$ \\
\hline \multicolumn{2}{|l|}{ Anti-diabetic medication, $N(\%)$} \\
\hline Monotherapy & $75(3.7)$ \\
\hline Dual therapy & $551(27.4)$ \\
\hline Triple therapy or more & $1004(50.0)$ \\
\hline Add on to insulin & $1025(18.8)$ \\
\hline \multicolumn{2}{|c|}{$\begin{array}{l}\text { Concomitant anti-diabetic medication, } \\
\qquad N(\%)\end{array}$} \\
\hline Metformin & $1813(90.3)$ \\
\hline
\end{tabular}

Table 1 continued

\begin{tabular}{ll}
\hline Baseline parameter & Value \\
\hline Sulfonylureas & $752(37.5)$ \\
Thiazolidinediones & $215(10.7)$ \\
DPP-4 inhibitor & $820(40.8)$ \\
Injection & $425(21.2)$ \\
\hline
\end{tabular}

Data for continuous variables are expressed as mean $\pm s$ tandard deviation (SD) and for categorical variables as number (percent)

$I Q R$ interquartile range, $B M I$ body mass index, SBP systolic blood pressure, $D B P$ diastolic blood pressure, $F P G$ fasting plasma glucose, $P P G$ postprandial glucose, $H b A 1 c$ glycated hemoglobin

$7.5 \pm 1.2 \%, \quad$ absolute $\Delta-0.6 \pm 1.2 \%$ for HbA1c; from $166.2 \pm 48.8$ to $139.6 \pm 38.9 \mathrm{mg} /$ dl, absolute $\Delta-26.6 \pm 49.8$ for FPG; from $233.1 \pm 80.4$ to $189.9 \pm 66.4 \mathrm{mg} / \mathrm{dl}$, absolute $\Delta$ $-43.1 \pm 84.9 \mathrm{mg} / \mathrm{dl}$ for PPG; Table 2). Body weight and BMI were also significantly $(P<0.001)$ decreased after dapagliflozin treatment (from $75.8 \pm 15.0$ to $73.9 \pm 14.4 \mathrm{~kg}$, absolute $\Delta-2.3 \pm 2.6 \mathrm{~kg}$ for body weight; from $28.1 \pm 4.4$ to $27.6 \pm 4.2 \mathrm{~kg} / \mathrm{m}^{2}$, absolute $\Delta$ $-0.9 \pm 1.0 \mathrm{~kg} / \mathrm{m}^{2}$ for BMI). More decreases in glycemic and anthropometric parameters were observed after 24 weeks of treatment with dapagliflozin. Although blood pressure was in the recommended cutoff range for T2D at baseline, gradual significant declines $(P<0.001)$ in both systolic and diastolic blood pressure were observed during dapagliflozin treatment $(128.1 \pm 14.2$ to $123.2 \pm 13.7 \mathrm{mmHg}$ for systolic blood pressure; $77.8 \pm 10.3$ to $75.3 \pm 10.3 \mathrm{mmHg}$ for diastolic blood pressure).

\section{Clinical Determinants Associated with Dapagliflozin Response}

Since dapagliflozin treatment was effective in lowering body weight and glucose levels, we explored the associations among relative changes in HbA1c, baseline HbA1c, and body weight. The results shown in Fig. 2a indicate a strong 
Table 2 Changes of clinical parameters after dapagliflozin treatment for 12 and 24 weeks

\begin{tabular}{lcllccl}
\hline Parameter & 12 Weeks & Change & P value & 24 Weeks & Change & P value \\
\hline HbAlc, \% (SD) & $7.5(1.2)$ & $-0.6(1.2)$ & $<0.001$ & $7.2(1.0)$ & $-0.8(1.2)$ & $<0.001$ \\
FPG, mg/dl (SD) & $139.6(38.9)$ & $-26.6(49.8)$ & $<0.001$ & $133.9(31.0)$ & $-27.0(48.8)$ & $<0.001$ \\
PPG, mg/dl (SD) & $189.9(66.4)$ & $-43.1(84.9)$ & $<0.001$ & $172.1(56.0)$ & $-51.6(92.6)$ & $<0.001$ \\
BMI, kg/m ${ }^{2}(\mathrm{SD})$ & $27.6(4.2)$ & $-0.9(1.0)$ & $<0.001$ & $27.6(4.7)$ & $-1.0(1.3)$ & $<0.001$ \\
Weight, kg (SD) & $73.9(14.4)$ & $-2.3(2.6)$ & $<0.001$ & $74.7(16.0)$ & $-2.7(3.6)$ & $<0.001$ \\
SBP, mmHg (SD) & $125.8(13.2)$ & $-3.1(14.5)$ & $<0.001$ & $123.2(13.7)$ & $-2.3(13.9)$ & $<0.001$ \\
DBP, mmHg (SD) & $76.7(9.8)$ & $-1.6(10.2)$ & $<0.001$ & $75.3(10.3)$ & $-1.1(10.6)$ & $<0.001$ \\
\hline
\end{tabular}

Data are presented as mean \pm standard deviations

$H b A 1 c$ glycated hemoglobin, FPG fasting plasma glucose, $P P G$ postprandial glucose, $B M I$ body mass index, $S B P$ systolic blood pressure, $D B P$ diastolic blood pressure

relationship between the baseline $\mathrm{HbA1c}$ value and dapagliflozin-induced response in HbA1c change $\left(R^{2}=0.27, \quad P<0.001\right)$. The relative HbA1c change was proportional to the baseline HbA1c (Fig. 2b). However, the correlation between baseline BMI and HbA1c change was not statistically significant $\left(R^{2}=0.001\right.$, $P=0.295$, Fig. 2c). Of the 1180 patients with both $\mathrm{HbA} 1 \mathrm{c}$ and body weight information at baseline and follow-up, 886 (75.1\%) experienced HbA1c reduction and 799 (67.7\%) achieved both HbA1c lowering and body weight loss. More than a third of the patients in the 12-week dapagliflozin treatment group (761/ $2007 ; 37.9 \%)$ experienced $\geq 10 \%$ reduction in $\mathrm{HbA1c}$ and were categorized as responders. The optimal cutoff for baseline HbA1c in dapagliflozin response was $8.0 \%$ (Figure $\mathrm{S} 1$ ).

Clinical parameters associated with the dapagliflozin response were determined using logistic regression analysis. As shown in Table 3, younger age, male sex, short duration of T2D, higher glycemic parameters (HbA1c, FPG, and PPG), lower BMI, and lower serum creatinine with higher eGFR were linked with a dapagliflozin response. The responses to dapagliflozin were better when the SGLT2 inhibitor was used as an add-on therapy than when it was used as monotherapy. Baseline systolic blood pressure did not show any association.

\section{Renal Hyperfiltration, Adjusted Determinants, and Dapagliflozin Response}

Because lower serum creatinine with higher eGFR was linked with a dapagliflozin response, we stratified the data by eGFR and analyzed changes during the study period (Table 4). Individuals with higher baseline eGFR showed statistically significant $(P<0.001)$ responses in glucose reduction and body weight loss $(\Delta$ HbA1c 4.1\%, 95\% CI 3.1-5.2 for eGFR $<90 \mathrm{ml} /$ $\mathrm{min} / 1.73 \mathrm{~m}^{2} ; \Delta \mathrm{HbA} 1 \mathrm{c} 7.6 \%, 95 \%$ CI $6.9-8.4$ for eGFR $\geq 90$ and $<120 \mathrm{ml} / \mathrm{min} / 1.73 \mathrm{~m}^{2} ; \Delta \mathrm{HbA} 1 \mathrm{c}$ $9.5 \%, 95 \%$ CI $6.8-12.3$ for eGFR $\geq 120 \mathrm{ml} / \mathrm{min} /$ $1.73 \mathrm{~m}^{2}$ ). However, the changes in systolic and diastolic blood pressure were not different among eGFR groups $(P=0.457$ for systolic blood pressure, $P=0.548$ for diastolic blood pressure).

To assess the independent clinical determinants related to dapagliflozin response, we performed multiple logistic regression analyses (Table 3). Individuals with an early T2D course (adjusted OR 0.46 95\% CI 0.30-0.69), higher HbA1c at baseline (adjusted OR 3.12, 95\% CI 2.48-3.92), and dapagliflozin use as an add-on therapy (adjusted OR 2.71, 95\% CI 1.72-4.27) still showed statistical significance in dapagliflozin responses (all $P<0.05$ ). In contrast, young age, male sex, hyperfiltration, and low BMI (BMI $<25 \mathrm{~kg} / \mathrm{m}^{2}$ ) showed only an 

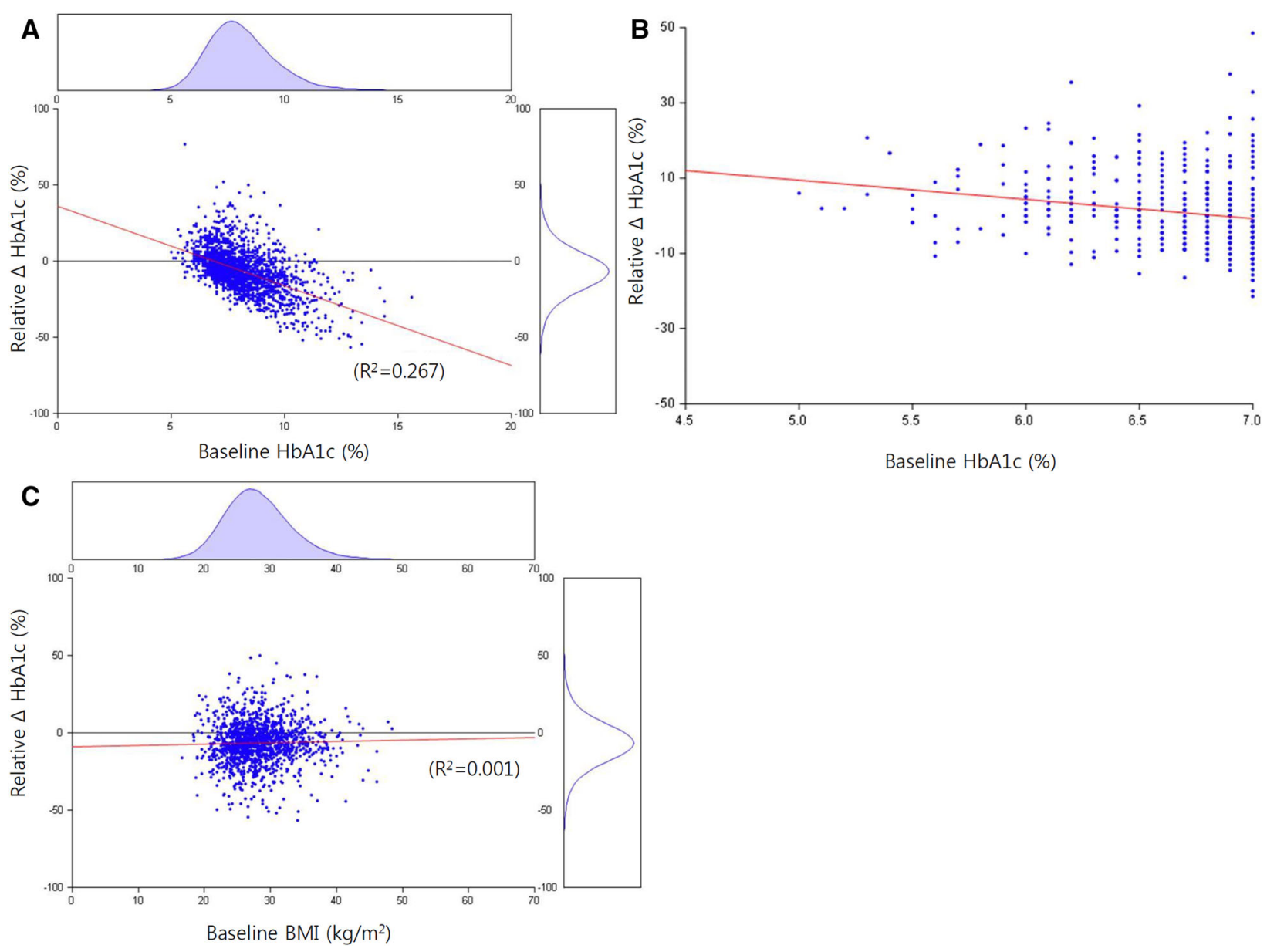

Fig. 2 Correlation among baseline glycated hemoglobin, body mass index, and change in glycated hemoglobin after 12-week treatment with dapagliflozin. a Correlation between baseline glycated hemoglobin (HbAlc) and relative $\triangle \mathrm{HbAlc}$ after 12 -week treatment with

increased trend in response to dapagliflozin treatment that was not statistically significant.

\section{Safety}

During the dapagliflozin treatment period, $26.3 \%$ (886 out of 3371) reported adverse events, and $57(1.6 \%)$ serious adverse events occurred (Table S1). Among the patients with serious adverse events, 52 were reported as completely recovered, 2 were in recovery, and 3 recovered with sequalae (traffic accident, right hand fracture, spontaneous brain hemorrhage). In addition, 127 (3.8\%) patients reported renal

dapagliflozin. $\mathbf{b}$ Distribution of patients for the relation between relative $\triangle \mathrm{HbA1c}$ and baseline HbAlc. c Correlation between baseline body mass index (BMI) and relative $\triangle$ HbAlc after 12-week treatment with dapagliflozin

and urinary disorders (polyuria, nocturia, and pollakiuria without progression to chronic kidney disease), and 45 (1.3\%) had genital infections, including 7 cases of urinary tract infection, cystitis, and vaginitis. Vascular-related side effects included one case of orthostatic hypotension and one case of hypotension. There was no neoplasm development or diagnosis during the study period.

\section{DISCUSSION}

Three significant findings emerged from this study to discern the characteristics of 
Table 3 Logistic regression analysis to determine variables associated with dapagliflozin responders (relative HbA1c reduction $\geq 10 \%)$ in 12 weeks

\begin{tabular}{|c|c|c|c|}
\hline Variable & Odds ratio & $95 \% \mathrm{CI}$ & $P$ value \\
\hline \multicolumn{4}{|l|}{ Univariate } \\
\hline Age, years & 0.99 & $0.98-0.99$ & 0.003 \\
\hline Sex, male & 1.26 & $1.06-1.51$ & 0.011 \\
\hline Diabetes duration ( $\geq 24$ months) & 0.41 & $0.32-0.54$ & $<0.001$ \\
\hline Baseline BMI, $\mathrm{kg} / \mathrm{m}^{2}$ & 0.97 & $0.95-0.99$ & 0.013 \\
\hline Baseline SBP, mmHg & 1.01 & $1.00-1.01$ & 0.140 \\
\hline Baseline HbAlc, \% & 2.31 & $2.11-2.54$ & $<0.001$ \\
\hline Baseline FPG, mg/dl & 1.01 & $1.01-1.02$ & $<0.001$ \\
\hline Baseline PPG, mg/dl & 1.01 & $1.01-1.01$ & $<0.001$ \\
\hline Baseline creatinine, mg/dl & 0.56 & $0.33-0.95$ & 0.031 \\
\hline Baseline eGFR & 1.01 & $1.01-1.02$ & $<0.001$ \\
\hline Anti-diabetic medication, add-on & 2.49 & $2.07-3.01$ & $<0.001$ \\
\hline \multicolumn{4}{|l|}{ Multivariate } \\
\hline Age ( $\geq 50$ years $)$ & 0.71 & $0.47-1.09$ & 0.116 \\
\hline Sex, male & 1.19 & $0.80-1.78$ & 0.385 \\
\hline Diabetes duration ( $\geq 24$ months) & 0.46 & $0.30-0.69$ & 0.001 \\
\hline Baseline BMI $\left(\geq 25 \mathrm{~kg} / \mathrm{m}^{2}\right)$ & 0.72 & $0.45-1.16$ & 0.175 \\
\hline Baseline HbAlc, \% & 3.12 & $2.48-3.92$ & $<0.001$ \\
\hline Baseline eGFR, $\left(\geq 90 \mathrm{ml} / \mathrm{min} / \mathrm{m}^{2}\right)$ & 0.98 & $0.63-1.53$ & 0.923 \\
\hline Anti-diabetic medication, add-on & 2.71 & $1.72-4.27$ & $<0.001$ \\
\hline
\end{tabular}

$B M I$ body mass index, $S B P$ systolic blood pressure, $H b A I c$ glycated hemoglobin, $F P G$ fasting plasma glucose, $P P G$ postprandial glucose, $e G F R$ estimated glomerular filtration rate

dapagliflozin responders and associations between the glucose-lowering effectiveness of dapagliflozin and other clinical parameters in an actual clinical setting. First, reductions in HbA1c and BMI were observed among dapagliflozin-treated patients in a real-world setting. Second, T2D patients with early diabetes course, higher baseline HbA1c, and dapagliflozin treatment as an add-on therapy were more likely to have significant clinical responses to dapagliflozin. Third, lower serum creatinine and higher eGFR were associated with a greater response to dapagliflozin treatment.
Considering the glucose-lowering effectiveness of dapagliflozin, $46.6 \%$ of the participants were switched to dapagliflozin from another medication and $53.3 \%$ participants had dapagliflozin added on to their treatment at baseline. Overall, 886 of 1180 (75.1\%) enrolled patients experienced HbA1c reductions. The overall change of HbA1c over 24 weeks in the actual clinical practice world was $-0.8 \%$, which is similar to the average $0.9 \% \mathrm{HbA} 1 \mathrm{c}$ reduction of dapagliflozin monotherapy in a previous RCT study [7]. This result indicates that it could be clinically relevant for a 


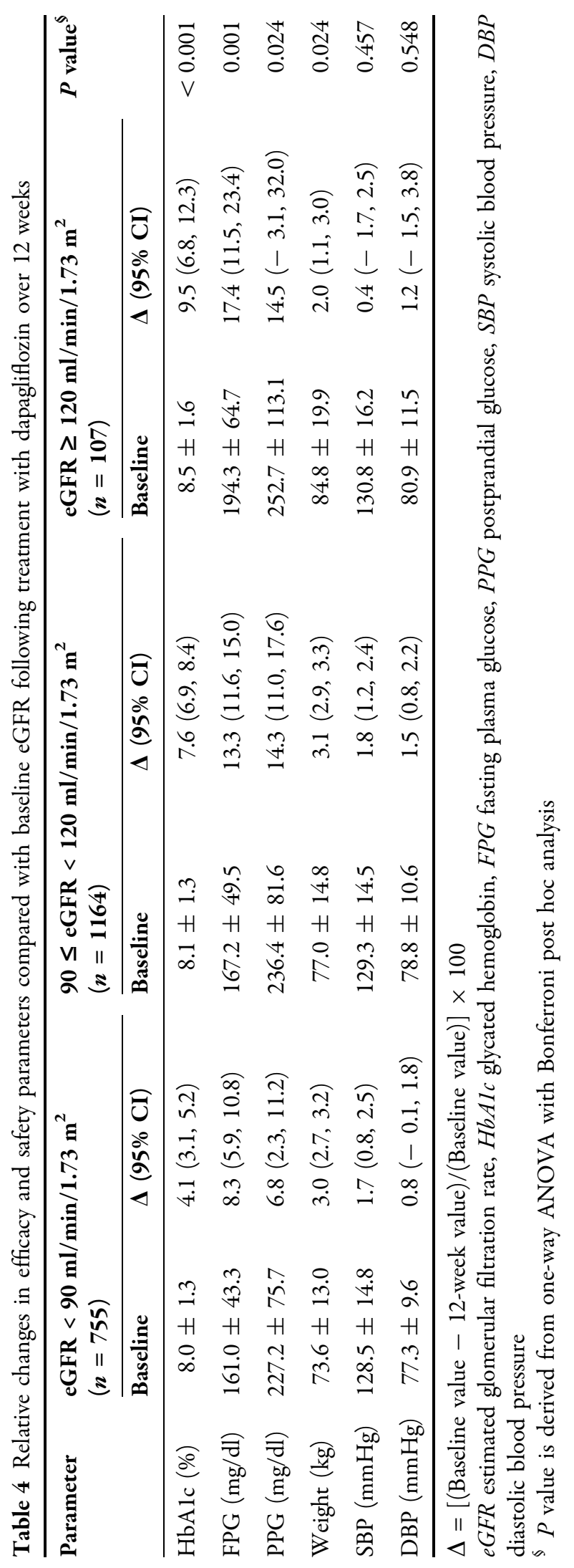


physician to prescribe an SGLT2 inhibitor for T2D patients with some modifying factors that would elicit the maximal effect of the SGLT2 inhibitor.

Characteristics of SGLT2 inhibitor responders in this study include higher baseline HbA1c and preserved eGFR reflected by young age and a shorter duration of diabetes. These characteristics have been noted in previous studies. A retrospective report showed that only higher baseline HbA1c and preserved eGFR (>90 ml/ $\min / 1.73 \mathrm{~m}^{2}$ ) had positive correlations with glucose-lowering effectiveness [14]. Moreover, in a pooled analysis report, the HbA1c-lowering effect was decreased as eGFR declined [15]. A Japanese pilot study demonstrated that the renal threshold of the glucose slope was significantly steeper in young ( $\leq 40$ years) compared with older adults ( $>40$ years) suggesting that the large response of urinary glucose excretion in the young accounts for the better response of SGLT2 inhibitors in young patients [16].

Similar to previous studies $[14,15,17]$, a significant association between body weight change and glucose reduction was not observed in our study. Because SGLT2 inhibitors impede glucose reabsorption from the proximal renal tubules, dapagliflozin induces a complimentary increase in urinary glucose excretion [17]. There are different results among numerous studies, but whether the extent of urine glucose excretion would drive the blood glucose decrease in a dependent manner has not been fully investigated. In a Japanese clinical trial, the young age group ( $\leq 40$ years) showed a better effectiveness in glycemic control with a larger increase in urine glucose excretion [16]. However, another study reported that a larger urinary glucose excretion did not reflect a better $\mathrm{HbA1c}$ reduction [18]. As expected, these results suggest that the insulin-independent mechanism for the glucose-lowering effect of an SGLT2 inhibitor is not simple.

Higher eGFR was associated with dapagliflozin responders in this study. Interestingly, there are reports that glomerular hyperfiltration might predict the development of diabetes and also be associated with obesity, hypertension, and even sarcopenia [19-22]. In a healthy and non-diabetic cohort, renal hyperfiltration was correlated with postprandial glucose metabolism and insulin sensitivity, whereas eGFR was not related to BMI and blood pressure [23]. Furthermore, lower insulin sensitivity was exhibited in a renal hyperfiltration group compared with controls [22]. Based on these previous studies regarding the correlation of renal hyperfiltration with insulin resistance and the association of increased eGFR with the glucoselowering effects of dapagliflozin, we postulate that the SGLT2 inhibitors could reduce glomerular hyperfiltration and $\mathrm{HbA1c}$ in an insulin-independent manner for T2D patients with early diabetes and renal hyperfiltration. In addition, improved glycemic status due to SGLT2 inhibitor therapy might attenuate insulin resistance or enhance inulin sensitivity rather than modify insulin secretion. Indeed, in the context of glucometabolic homeostasis, a hyperinsulinemic euglycemic clamp study demonstrated that SGLT2 inhibitor therapy reduced insulin secretion and improved insulin sensitivity of glucose uptake [24].

This study has some limitations. First, due to the lack of information attributable to the nature of a PMS, we cannot assert a direct association between renal hyperfiltration and changes in those parameters after dapagliflozin treatment. Second, we did not account for the potential effect of other medications on renal hyperfiltration, including the renin-angiotensin-aldosterone system. Third, we did not measure the uric acid level in the current study. Several studies have consistently reported that SGLT2 inhibitor treatment decreases serum uric acid by altering uric acid transport activity [25-28]. It has been hypothesized that the cardio-protective effects of SGLT2 inhibitors arise from uric acid-lowering effects [29]. In addition, the uric acid-lowering effects are related to the HbA1c reduction [27]. Fourth, even though missing data were imputed using the last observation carried forward method, only 1180 out of 2007 patients had all values for HbA1c and body weight at both baseline and 12-week follow-up, which was a limitation for data analysis. Fourth, even though missing data were imputed using the last observation carried forward method, only 1180 out of 2007 patients had all values for HbA1c and body weight at 
both baseline and 12-week follow up, which was a limitation for data analysis. Finally, as the original purpose of the current study was postmarketing surveillance, we only assessed the effect of dapagliflozin up to 24 weeks. Further studies are warranted for the long-term effects of dapagliflozin. Despite these limitations, our study has the advantages of utilizing data from a large, population-based study and of exploring the characteristics of Korean dapagliflozin responders in a real-world setting.

\section{CONCLUSIONS}

This nationwide survey of a Korean population demonstrated that a better response to dapagliflozin therapy is expected in T2D patients who are in a relatively early diabetes course with uncontrolled hyperglycemia. In addition, renal hyperfiltration might be associated with higher dapagliflozin response. Further prospective, well-designed, longitudinal studies with sufficient laboratory resources are warranted to establish the clinical factors that indicate the use of an SGLT2 inhibitor and to identify optimal combinations of an SGLT2 inhibitor with other anti-diabetic medications.

\section{ACKNOWLEDGEMENTS}

Funding. Sponsorship for this study and article processing charges were funded by Astra Zeneca.

Authorship. All named authors meet the International Committee of Medical Journal Editors (ICMJE) criteria for authorship for this article, take responsibility for the integrity of the work as a whole, and have given their approval for this version to be published.

Prior Presentation. The data were partially presented at the 17th Congress of the International Diabetes Federation 5-8 December 2017 (abstract).
Disclosures. Eugene Han, Jae Hyeon Kim, Woo Je Lee, and Byung-Wan Lee have nothing to disclose. Ari Kim is an employee of AstraZeneca. Seung Jae Lee is a former employee of AstraZeneca. Je-Yon Kim is a former employee of AstraZeneca.

Compliance with Ethics Guidelines. This study was conducted in compliance with the ethical principles of the "Basic Standards for the Re-examination of New Medicines" published by the Ministry of Food and Drug Safety in Korea. Approval from the Institutional Review Board was obtained according to the rules of each institution as required for a PMS. All procedures followed were in accordance with the ethical standards of the responsible committee on human experimentation (institutional and national) and with the Helsinki Declaration of 1964, as revised in 2013. Informed consent was obtained from all patients for being included in the study.

Data Availability. The data sets during and/ or analyzed during the current study are available from the corresponding author on reasonable request.

Open Access. This article is distributed under the terms of the Creative Commons Attribution-NonCommercial 4.0 International License (http://creativecommons.org/licenses/ by-nc/4.0/), which permits any noncommercial use, distribution, and reproduction in any medium, provided you give appropriate credit to the original author(s) and the source, provide a link to the Creative Commons license, and indicate if changes were made.

\section{REFERENCES}

1. Abdul-Ghani MA, Norton L, Defronzo RA. Role of sodium-glucose cotransporter 2 (SGLT 2) inhibitors in the treatment of type 2 diabetes. Endocr Rev. 2011;32(4):515-31.

2. Jung CH, Jang JE, Park JY. A novel therapeutic agent for type 2 diabetes mellitus: SGLT2 inhibitor. Diabetes Metab J. 2014;38(4):261-73. 
3. Min KW, Ku BJ, Lee JH, Kim MS, Ahn KJ, Lee MK, et al. Addition of Ipragliflozin to metformin treatment in Korean patients with type 2 diabetes mellitus: subgroup analysis of a phase 3 trial. Diabetes Metab J. 2017;41(2):135-45.

4. Zinman B, Wanner C, Lachin JM, Fitchett D, Bluhmki E, Hantel S, et al. Empagliflozin, cardiovascular outcomes, and mortality in type 2 diabetes. N Engl J Med. 2015;373(22):2117-28.

5. Bailey CJ, Gross JL, Pieters A, Bastien A, List JF. Effect of dapagliflozin in patients with type 2 diabetes who have inadequate glycaemic control with metformin: a randomised, double-blind, placebocontrolled trial. Lancet. 2010;375(9733): 2223-33.

6. Bolinder J, Ljunggren $\mathrm{O}$, Kullberg J, Johansson L, Wilding J, Langkilde AM, et al. Effects of dapagliflozin on body weight, total fat mass, and regional adipose tissue distribution in patients with type 2 diabetes mellitus with inadequate glycemic control on metformin. J Clin Endocrinol Metab. 2012;97(3):1020-31.

7. Ferrannini E, Ramos SJ, Salsali A, Tang W, List JF. Dapagliflozin monotherapy in type 2 diabetic patients with inadequate glycemic control by diet and exercise: a randomized, double-blind, placebocontrolled, phase 3 trial. Diabetes Care. 2010;33(10):2217-24.

8. Rosenstock J, Vico M, Wei L, Salsali A, List JF. Effects of dapagliflozin, an SGLT2 inhibitor, on $\operatorname{HbA}(1 \mathrm{c})$, body weight, and hypoglycemia risk in patients with type 2 diabetes inadequately controlled on pioglitazone monotherapy. Diabetes Care. 2012;35(7):1473-8.

9. Henry RR, Murray AV, Marmolejo MH, Hennicken D, Ptaszynska A, List JF. Dapagliflozin, metformin $\mathrm{XR}$, or both: initial pharmacotherapy for type 2 diabetes, a randomised controlled trial. Int J Clin Pract. 2012;66(5):446-56.

10. Levey AS, Stevens LA, Schmid CH, Zhang YL, Castro AF 3rd, Feldman HI, et al. A new equation to estimate glomerular filtration rate. Ann Intern Med. 2009;150(9):604-12.

11. Han E, Park HS, Kwon O, Choe EY, Wang HJ, Lee $\mathrm{YH}$, et al. A genetic variant in GLP1R is associated with response to DPP-4 inhibitors in patients with type 2 diabetes. Medicine (Baltim). 2016;95(44): e5155.

12. International Council for Harmonisation of technical requirements for registration of pharmaceuticals for human use. Medical Dictionary for Regulatory Activities Version 181, Geneva. 2015.
13. Kim CS, Ko SH, Kwon HS, Kim NH, Kim JH, Lim S, et al. Prevalence, awareness, and management of obesity in Korea: data from the Korea national health and nutrition examination survey (1998-2011). Diabetes Metab J. 2014;38(1):35-43.

14. Lee JY, Kim G, Kim SR, Lee YH, Lee BW, Cha BS, et al. Clinical parameters affecting dapagliflozin response in patients with type 2 diabetes. Diabetes Metab. 2017;43(2):191-4.

15. Petrykiv S, Sjostrom CD, Greasley PJ, Xu J, Persson F, Heerspink HJL. Differential effects of dapagliflozin on cardiovascular risk factors at varying degrees of renal function. Clin J Am Soc Nephrol. 2017;12(5):751-9.

16. Nakamura $Y$, Nagai $Y$, Terashima $Y$, Nishine A, Ishii $\mathrm{S}$, Kato $\mathrm{H}$, et al. Better response to the SGLT2 inhibitor dapagliflozin in young adults with type 2 diabetes. Expert Opin Pharmacother. 2015;16(17): 2553-9.

17. Zhang L, Feng Y, List J, Kasichayanula S, Pfister M. Dapagliflozin treatment in patients with different stages of type 2 diabetes mellitus: effects on glycaemic control and body weight. Diabetes Obes Metab. 2010;12(6):510-6.

18. Kim SR, Lee YH, Kang ES, Cha BS, Lee BW. The relationship between Increases in morning spot urinary glucose excretion and decreases in HbA1C in patients with type 2 diabetes after taking an SGLT2 inhibitor: a retrospective, longitudinal study. Diabetes Ther. 2017;8(3):601-9.

19. Han E, Lee YH, Lee BW, Kang ES, Cha BS. Pre-sarcopenia is associated with renal hyperfiltration independent of obesity or insulin resistance: Nationwide Surveys (KNHANES 2008-2011). Medicine (Baltim). 2017;96(26):e7165.

20. Helal I, Fick-Brosnahan GM, Reed-Gitomer B, Schrier RW. Glomerular hyperfiltration: definitions, mechanisms and clinical implications. Nat Rev Nephrol. 2012;8(5):293-300.

21. Sim EH, Lee HW, Choi HJ, Jeong DW, Son SM, Kang $\mathrm{YH}$. The association of serum cystatin $\mathrm{C}$ with glycosylated hemoglobin in Korean adults. Diabetes Metab J. 2016;40(1):62-9.

22. Tomaszewski M, Charchar FJ, Maric C, McClure J, Crawford L, Grzeszczak W, et al. Glomerular hyperfiltration: a new marker of metabolic risk. Kidney Int. 2007;71(8):816-21.

23. Naderpoor N, Lyons JG, Mousa A, Ranasinha S, Courten MP, Soldatos G, et al. Higher glomerular filtration rate is related to insulin resistance but not to obesity in a predominantly obese non-diabetic cohort. Sci Rep. 2017;7:45522. 
24. Ferrannini E, Muscelli E, Frascerra S, Baldi S, Mari A, Heise T, et al. Metabolic response to sodium-glucose cotransporter 2 inhibition in type 2 diabetic patients. J Clin Invest. 2014;124(2):499-508.

25. Davies MJ, Trujillo A, Vijapurkar U, Damaraju CV, Meininger G. Effect of canagliflozin on serum uric acid in patients with type 2 diabetes mellitus. Diabetes Obes Metab. 2015;17(4):426-9.

26. Chino Y, Samukawa Y, Sakai S, Nakai Y, Yamaguchi J, Nakanishi T, et al. SGLT2 inhibitor lowers serum uric acid through alteration of uric acid transport activity in renal tubule by increased glycosuria. Biopharm Drug Dispos. 2014;35(7):391-404.

27. Ouchi M, Oba K, Kaku K, Suganami H, Yoshida A, Fukunaka Y, et al. Uric acid lowering in relation to
HbA1c reductions with the SGLT2 inhibitor tofogliflozin. Diabetes Obes Metab. 2018;20(4):1061-5.

28. Zhao Y, Xu L, Tian D, Xia P, Zheng H, Wang L, et al. Effects of sodium-glucose co-transporter 2 (SGLT2) inhibitors on serum uric acid level: a meta-analysis of randomized controlled trials. Diabetes Obes Metab. 2018;20(2):458-62.

29. Zoppini G, Targher G, Negri C, Stoico V, Perrone F, Muggeo $\mathrm{M}$, et al. Elevated serum uric acid concentrations independently predict cardiovascular mortality in type 2 diabetic patients. Diabetes Care. 2009;32(9):1716-20. 